\title{
Audio-guided self-hypnosis for reduction of claustrophobia during MR imaging: results of an observational 2-group study
}

\author{
Adriane E. Napp ${ }^{1} \cdot$ Torsten Diekhoff $^{1}$ (I) $\cdot$ Olf Stoiber $^{2} \cdot$ Judith Enders $^{1} \cdot$ Gerd Diederichs $^{3} \cdot$ Peter Martus $^{4}$. \\ Marc Dewey ${ }^{1}$
}

Received: 17 April 2020 / Revised: 22 December 2020 / Accepted: 15 March 2021 / Published online: 15 April 2021

(C) The Author(s) 2021

\begin{abstract}
Objectives To evaluate the influence of audio-guided self-hypnosis on claustrophobia in a high-risk cohort undergoing magnetic resonance (MR) imaging.

Methods In this prospective observational 2-group study, 55 patients (69\% female, mean age $53.6 \pm 13.9)$ used self-hypnosis directly before imaging. Claustrophobia included premature termination, sedation, and coping actions. The claustrophobia questionnaire (CLQ) was completed before self-hypnosis and after MR imaging. Results were compared to a control cohort of 89 patients examined on the same open MR scanner using logistic regression for multivariate analysis. Furthermore, patients were asked about their preferences for future imaging.

Results There was significantly fewer claustrophobia in the self-hypnosis group $(16 \% ; 9 / 55)$, compared with the control group $(43 \% ; 38 / 89$; odds ratio $.14 ; p=.001)$. Self-hypnosis patients also needed less sedation ( $2 \%$ vs $16 \% ; 1 / 55$ vs $14 / 89$; odds ratio .1; $p=.008)$ and non-sedation coping actions ( $13 \%$ vs $28 \% ; 7 / 55$ vs $25 / 89$; odds ratio $.3 ; p=.02$ ). Self-hypnosis did not influence the CLQ results measured before and after MR imaging $(p=.79)$. Self-hypnosis reduced the frequency of claustrophobia in the subgroup of patients above an established CLQ cut-off of .33 from $47 \%(37 / 78)$ to $18 \%(9 / 49 ; p=.002)$. In the subgroup below the CLQ cut-off of 0.33 , there were no significant differences ( $0 \%$ vs $9 \%, 0 / 6$ vs $1 / 11 ; p=1.0)$. Most patients $(67 \% ; 35 / 52)$ preferred self-hypnosis for future MR examinations.

Conclusions Self-hypnosis reduced claustrophobia in high-risk patients undergoing imaging in an open MR scanner and might reduce the need for sedation and non-sedation coping actions.

Key Points

- Forty percent of the patients at high risk for claustrophobia may also experience a claustrophobic event in an open MR scanner.

- Self-hypnosis while listening to an audio in the waiting room before the examination may reduce claustrophobic events in over $50 \%$ of patients with high risk for claustrophobia.

- Self-hypnosis may also reduce the need for sedation and other time-consuming non-sedation coping actions and is preferred by high-risk patients for future examinations.
\end{abstract}

Keywords Phobic disorders $\cdot$ Anxiety $\cdot$ Magnetic resonance imaging $\cdot$ Hypnosis

Adriane E. Napp and Torsten Diekhoff contributed equally to this work.

Torsten Diekhoff torsten.diekhoff@charite.de

Marc Dewey dewey@charite.de

1 Department of Radiology, Charité - Universitätsmedizin Berlin Campus Mitte, Humboldt-Universität zu Berlin, Freie Universität Berlin, Charité Platz 1, 10117 Berlin, Germany
2 Hypnovita, Academy of Hypnotic Medical Arts, Josephsburgstraße 33, 81673 Munich, Germany

3 Department of Radiology, Charité - Universitätsmedizin Berlin Campus Vircho-Klinikum, Humboldt-Universität zu Berlin, Freie Universität Berlin, Augustenburger Platz 1, 13353 Berlin, Germany

4 Clinical Epidemiology and Applied Biometry, Universitätsklinikum Tübingen, Silcherstraße 5, 72076 Tübingen, Germany 


$\begin{array}{ll}\text { Abbreviations } \\ \text { AUROC } & \begin{array}{l}\text { Area under the receiver operation characteristic } \\ \text { curve }\end{array} \\ \text { CLQ } & \text { Claustrophobia questionnaire } \\ \text { ICD } & \text { Implantable cardioverter-defibrillators } \\ \text { LR } & \text { Likelihood ratio } \\ \text { MR } & \text { Magnetic resonance } \\ \text { NPV } & \text { Negative predictive value } \\ \text { PPV } & \text { Positive predictive value }\end{array}$

\section{Introduction}

Up to $10 \%$ of patients cannot undergo magnetic resonance (MR) imaging due to severe claustrophobia triggered by the small diameter of the MR tube or loud noises [1,2]. In view of recent studies indicating that, with appropriate precautions, pacemakers and implantable cardioverter-defibrillators (ICD) no longer preclude MR examinations [3, 4], severe claustrophobia is the main remaining relative contraindication to MR imaging. Patients may experience anxiety when encountering MR imaging or being inside the MR tube during the examination [5], especially if the procedure is unknown to them and they lack information about what to expect [6]. Claustrophobic events are common but their prevalence varies with patients' socioeconomic background and medical history [7]. Dealing with claustrophobic patients is a challenge for medical staff $[8,9]$. Besides the feeling of confinement, the noise of the machine during the scan has been identified as a major factor inducing fear and discomfort $[2,10]$. However, there are many factors that influence claustrophobia in general and during MR imaging in particular [11].

Distress during acquisition may lead to premature termination of the scan or reduce image quality due to motion artifacts, impeding proper interpretation of images [6]. Therefore, many patients need conscious sedation to complete the examination [12,13], which requires monitoring of vital functions and exposes patients to the risk of severe complications [14]. Furthermore, medical sedation requires an experienced operator to ensure maximum efficiency, which is not available in all hospitals or outpatient centers [15]. To avoid medications or to at least reduce their dose and hence the risks, several alternative strategies have been proposed and investigated to reduce patients' fear during MR imaging [16-18]. Hypnosis and relaxation techniques have been found to reduce patients' anxiety and the need for medication during medical procedures [19-22]. Hypnosis may be considered as a modified state of consciousness that emphasizes attention, concentration, and the letting go of thoughts and is characterized by mental relaxation, altered perception of the environment, and disengagement of the discursive and critical analytical reasoning [23]. While many hypnotherapists stress that hypnotic suggestions have to be internalized before being effective, the definition of self-hypnosis remains vague. For this manuscript, the hypnotic intervention was defined as selfhypnosis because a sufficient amount of effort originated from the patient and no hypnotherapist was present. From an economic point of view, strategies that do not prolong the examination or preparation are of particular interest here as MR imaging is already expensive compared to other imaging modalities. Therefore, relaxation techniques or so-called selfhypnosis may be an interesting and effective strategy to reduce the need for oral or intravenous sedation, while at the same time keeping the patient comfortable through the examination.

We hypothesize that audio-guided self-hypnosis before MR imaging can reduce claustrophobic events in patients at an increased risk of claustrophobia during MR imaging in an open scanner. Therefore, the aim of our study was to evaluate self-hypnosis in patients with an increased risk of anxiety and claustrophobia compared to a control group of patients who also presented with this increased risk but were examined on the same MR scanner without self-hypnosis. The focus of the analysis was on the frequency of incomplete examinations, use of medical sedation, and non-sedation coping strategies. We furthermore assessed patients' satisfaction with and acceptance of self-hypnosis as preparation for their examination.

\section{Materials and methods}

\section{Ethics approval and informed consent}

The study was approved by the local institutional review board (EA1/237/12). All patients in the intervention group gave written informed consent. No written informed consent was required and waived by the institutional review board from patients in the control cohort, who were only asked to complete the German version of the validated Englishlanguage claustrophobia questionnaire (CLQ) before the MR examination [24]. During recruitment, we noted a difference in gender distribution compared with the control cohort. Therefore, an amendment to the original ethics approval was granted to investigate more male patients.

\section{Study design}

The prospective intervention cohort included patients who were scheduled to undergo an MR examination on our 1.0-T open MR scanner (Panorama, Philips Healthcare). These were mostly patients with known claustrophobia or patients with discomfort during previous examinations for whom the referring physicians or the patients themselves asked for an examination on an open MR system. Thus, we expected a high-risk 
cohort. All other inclusion and exclusion criteria were the same as for the control group [11]. Exclusion criteria were referrals from the intensive care unit, severe emergencies, general poor health, severe psychological disorders, invasive procedures during MR imaging (e.g., biopsies), age under 18, and examinations specifically scheduled for other studies. Furthermore, patients were excluded if they were not able to answer the questions of the claustrophobia questionnaire (CLQ) prior to the examination. Patients were included in the analysis if they remained in the MR scanner for at least $20 \mathrm{~min}$, even if their examinations could not be completed because of technical problems. These examinations were not counted as premature terminations due to claustrophobia. If patients underwent two examinations with self-hypnosis, only the first examination and the corresponding questionnaire data were used for this analysis

At a time during the study, patients in both cohorts (intervention and control) were offered and granted medical sedation or coping actions to make them feel confident to try selfhypnosis. This approach enabled an intention-to-treat analysis.

\section{Self-hypnosis intervention}

A hypnotherapist (OS) informed of the specific needs of patients in the MR scanner created a self-hypnosis script which included self-empowering and metaphoric suggestions addressing the worries of patients inside an MR scanner and designed to reduce claustrophobic sensations during the examination (Appendix 1). While the script utilizes elements of several different hypnotic styles, it is mostly characterized by an indirect phrasing, much in the fashion of a modified socalled Ericksonian hypnosis. It was recorded as a 23-min audio file with background music and provided to the patient with an easy-to-use MP3 player and high-quality earphones (Bose AE2) while waiting and preparing for the examination. Patients and technicians could stop self-hypnosis at any time and had to stop for beginning the examination. During longer waiting periods, patients could restart the audio file. The exposure time to the self-hypnosis script was measured for each patient. There was no audio stimulation, especially no selfhypnosis, during the examination itself. Technicians were advised to handle the high-risk patients in the same way as their other patients.

\section{Study conduct}

All patients willing to participate completed the CLQ (Appendix 2) before the hypnotic intervention and after the MR examination. The CLQ consists of 26 questions picturing the patient in different situations and asking to which extent these induce fear - ranging from 0 (no fear) to 4 (maximum fear) [24]. A CLQ mean score was calculated by dividing the sum of all scores by the number of questions. Only questionnaires where all 26 questions were answered were considered. We recorded specific events during the examination that led to premature termination or a coping action and the reason why this event took place (e.g., claustrophobia or pain). An examination was also counted as prematurely terminated, if acquired images were non-diagnostic due to motion artifacts. Intravenous and oral sedation (using benzodiazepine) was used according to the guidelines of the American Society of Anesthesiology [25]. Non-sedation coping actions included a trial run with the patient in the scanner, a pause, an accompanying person in the scanner room, mirror glasses, or a specific, comforting conversation addressing issues of claustrophobia in the scanner. All events were protocoled, and it was explicitly assessed if the coping action was due to claustrophobia or possible other causes such as pain.

Besides completing the CLQ for a second time, patients were also asked to answer some questions after the examination describing their experience during the examination and self-hypnosis. The questions related to previous experiences with MR imaging and claustrophobic events, their subjective assessment of the usefulness of self-hypnosis, and if they would prefer further examinations with or without selfhypnosis (satisfaction questionnaire, Table 1).

\section{Definition of a claustrophobic event}

The occurrence of a claustrophobic event was assumed if the scan terminated prematurely or if claustrophobia required intravenous or oral sedation or a non-sedation coping action at any time before or during the examination to complete the scan. Claustrophobic events were ranked by severity, ranging from high (premature termination/non-diagnostic image quality), to moderate (intravenous or oral sedation), to low (nonsedation coping action). On the patient level, the most severe event counted for analysis - e.g., if a patient needed oral sedation and caused motion artifacts precluding diagnosis, the examination counted as prematurely terminated.

\section{Control group}

For comparison, we used the data from a cohort of patients with a similar risk of claustrophobia who were examined on the same 1.0-T open MR scanner. These patients also completed the CLQ before the examination but did not receive self-hypnosis. The study design has already been reported. However, the data of this specific cohort was not included by Napp et al in the analysis [11].

\section{Statistical analysis}

The statistical sample size was estimated by an expert statistician based on the hypothesis of a reduction of claustrophobic 
Table 1 Selected items and possible answers and number of answers of the satisfaction questionnaire. The satisfaction questionnaire was answered by 53 patients after magnetic resonance imaging ( $n$, number of patients). Not all patients completed all questions. Questions 1 and 1a do not refer to the study MR examination but to previous experiences.

\begin{tabular}{|c|c|c|c|c|c|}
\hline No & Item of the satisfaction questionnaire & Answers & & & \\
\hline 1 & $\begin{array}{l}\text { Did you ever experience claustrophobia before, during, } \\
\text { or after an MR examination previously? }\end{array}$ & $\begin{array}{l}\text { No } \\
(n=17)\end{array}$ & $\begin{array}{l}\text { Yes } \\
(n=28)\end{array}$ & $\begin{array}{l}\text { Several times } \\
(n=8)\end{array}$ & \\
\hline 1a & $\begin{array}{l}\text { Did you need support before or during previous } \\
\text { examinations? }\end{array}$ & $\begin{array}{l}\text { No } \\
(n=10) \\
\text { Test run } \\
(n=2)\end{array}$ & $\begin{array}{l}\text { Sedative injection } \\
(n=11) \\
\text { Pause } \\
(n=1)\end{array}$ & $\begin{array}{l}\begin{array}{l}\text { Oral sedation } \\
\text { tablet }\end{array} \\
(n=5) \\
\text { Prism glasses } \\
(n=4)\end{array}$ & $\begin{array}{l}\text { Prone position } \\
(n=0) \\
\text { Escort in scanner room } \\
(n=1)\end{array}$ \\
\hline 2 & How useful did you find self-hypnosis for this examination? & $\begin{array}{l}\text { Not at all } \\
(n=12)\end{array}$ & $\begin{array}{l}\text { A little } \\
(n=3)\end{array}$ & $\begin{array}{l}\text { Moderately } \\
(n=14)\end{array}$ & $\begin{array}{ll}\text { Useful } & \text { Very useful } \\
(n=13) & (n=11)\end{array}$ \\
\hline $3 \mathrm{a}$ & $\begin{array}{l}\text { Which kind of examination would you prefer for future } \\
\text { examinations on the open MR scanner? }\end{array}$ & $\begin{array}{l}\text { MR with self-hypnosis } \\
(n=35)\end{array}$ & \multicolumn{2}{|c|}{$\begin{array}{l}\text { MR without self-hypnosis } \\
(n=11)\end{array}$} & $\begin{array}{l}\text { I don’t know } \\
(n=6)\end{array}$ \\
\hline $3 b$ & $\begin{array}{l}\text { Which kind of examination would you prefer for } \\
\text { future examinations on a conventional MR scanner } \\
\text { with a normal bore? }\end{array}$ & $\begin{array}{l}\text { MR with self-hypnosis } \\
(n=29)\end{array}$ & \multicolumn{2}{|c|}{$\begin{array}{l}\text { MR without self-hypnosis } \\
(n=5)\end{array}$} & $\begin{array}{l}\text { I don't know } \\
(n=11)\end{array}$ \\
\hline
\end{tabular}

events from $43 \%$ (38 of 89) to $20 \%$ ( 9 of 46) by self-hypnosis. A two-tailed type 1 error of .05, a power of more than $80 \%$, and an expected drop-out rate of $10 \%$ yielded a total sample size of 51 patients. We performed a Mann-Whitney $U$-test to compare the self-hypnosis and control groups regarding age and CLQ score and a chi-square test for gender distribution. In logistic regression analyses with outcomes "claustrophobic events in total" and the subcategories "premature termination," "sedation," and "non-sedating coping actions," the effect of self-hypnosis was adjusted for age, gender, and CLQ. We performed a Wilcoxon matched-pairs signed-rank test to test for significant differences in CLQ mean values before and after self-hypnosis and the MR examination. We also compared the occurrence of events in patients with a CLQ below an established cut-off (.33 for all patients, Appendix 3) [11] and those above the cut-off using Fischer's exact test (below cut-off) and a chi-square test with Yates' correction (above cut-off). Furthermore, events in both groups were analyzed by type of examination. Statistical tests were executed using Prism (Version 6.0 for Mac, GraphPad Software Inc.) and SPSS (Version 23.0, IBMCorp). A $p$ value equal to or smaller than .05 was considered significant.

\section{Results}

During the study period, 87 examinations were conducted on the 1.0-T open MR scanner. Of these examinations, we included 58 examinations of patients willing to participate in the study, answered the CLQ prior to the examination, and used self-hypnosis for a mean of $23.3 \mathrm{~min}$ (20 to $40 \mathrm{~min}$ ). Three patients participated twice and, therefore, the last examination was excluded. Among the patients with repeated examinations, there was a 52-year-old woman with a relatively high CLQ (mean 2.1) who completed the first examination without need for sedation or non-sedation coping actions using selfhypnosis. However, for the second examination, she asked for additional oral sedation. Another patient was not offered selfhypnosis for the second examination because of time constraints. Despite being able to complete the first MR examination without any coping actions besides the interventional self-hypnosis, she needed intravenous sedation for the second scan. None of the patients were included in both the control and the intervention groups. Figure 1 shows a flow chart of study inclusion. Both groups had a mean CLQ value above the established cut-off of .33 [11]. The descriptive statistics of the intervention and the control group can be found in Table 2 and detailed information of anatomic regions in Table 3.

Use of audio-guided self-hypnosis reduced claustrophobic events from $43 \%(38 / 89)$ to $16 \%(9 / 55)$, the need for sedation from 16 to $2 \%(14 / 89$ vs $1 / 55)$, and non-sedation coping actions from 28 to $13 \%$ (25/89 vs 7/55, Table 2 and Fig. 2). Although the frequency of premature terminations was halved (3/55 vs 9/89), the difference was not statistically significant. The results of the multivariate logistic regression analysis are shown in Table 4 (univariate results are shown in Appendix 4). Whereas events in patients above the CLQ cut-off of .33 decreased significantly (9\% vs 47\%; $1 / 11$ vs 37/78; $p=.002$ ), no significant decrease was seen in patients below the cut-off ( $0 \%$ vs $9 \% ; 0 / 6$ vs $1 / 11 ; p=1.0)$.

In the intervention cohort, $11 \%$ of patients ( 6 of 55$)$ terminated the MR examination prematurely, $5 \%$ (3/55) due to claustrophobia, $4 \%(2 / 55)$ due to a technical problem with 
Table 2 Demography and predictors of self-hypnosis (intervention) in comparison to the control group. CLQ, claustrophobia questionnaire. Data are given in percentage (nominator/ denominator) or mean \pm standard deviation and (min-max)

\begin{tabular}{lllll}
\hline & Intervention $(n=55)$ & \multicolumn{2}{l}{ Controls $(n=89)$} \\
\hline Sex & & & & \\
Male & $31 \%$ & $(17 / 55)$ & $51 \%$ & $(45 / 89)$ \\
Female & $69 \%$ & $(38 / 55)$ & $49 \%$ & $(44 / 89)$ \\
Age & $53.6 \pm 13.9$ & {$[25-81]$} & $51.2 \pm 14.3$ & {$[19-82]$} \\
CLQ & $1.71 \pm 1.0$ & {$[0-3.8]$} & $1.51 \pm 1.0$ & {$[0-4.0]$} \\
Claustrophobic events (total) & $16 \%$ & $(9 / 55)$ & $43 \%$ & $(38 / 89)$ \\
Premature termination for claustrophobia & $5 \%$ & $(3 / 55)$ & $10 \%$ & $(9 / 89)$ \\
Sedation for claustrophobia & $2 \%$ & $(1 / 55)$ & $16 \%$ & $(14 / 89)$ \\
Coping for claustrophobia without sedation & $13 \%$ & $(7 / 55)$ & $28 \%$ & $(25 / 89)$ \\
\hline
\end{tabular}

the MR scanner, and $2 \%(1 / 55)$ due to a reduced general condition and pain. In the control group, there were $12 \%$ $(11 / 89)$ premature terminations, $10 \%(9 / 89)$ due to claustrophobia, and 2\% (2/89) for technical reasons.

There was no intravenous sedation in the intervention cohort $(0 / 55)$ compared to $7 \%(6 / 89)$ in the control group $(p=$ $.06)$. Oral sedation was required by $4 \%$ of the patients using self-hypnosis (2/55) — among them one who demanded sedation for pain and not for claustrophobia - compared to $9 \%$ $(8 / 89)$ in the control group $(p=.5)$.

Regarding non-sedation coping actions for claustrophobia, we observed extended conversations in 5\% (3 of 55) of the self-hypnosis group compared to $26 \%$ (23 of 89) in the control group $(p=.003), 4 \%(2 / 55)$ test runs (controls $12 \%(11 / 89), p$ $=.13), 4 \%(2 / 55)$ pauses (controls 3\% (3/89), $p=>.99), 2 \%(1$ of 55) escort person (controls $3 \%(3 / 89), p=.66)$, and no $(0 \%)$ other, unspecified coping action (controls $8 \%$; 7/89).

All patients completed all CLQ questions before the examination. Fifty patients answered all CLQ questions after the examination. There was no statistically significant difference in CLQ scores before and after the examination $(1.6 \pm .92 \mathrm{vs}$ $1.62 \pm 1.1 ; p=.79)$. All 53 patients who answered the satisfaction questionnaire had a previous MR examination, none of them in an open MR. The mean usefulness rating of selfhypnosis assigned by the patients was $2.23 \pm 1.35$, with scores ranging from 0 (not at all helpful) to 4 (very helpful) and 2 being neutral. However, $67 \%$ (35/52) of the patients preferred self-hypnosis on an open MR scanner. For examinations on a conventional MR imager, self-hypnosis was preferred by $64 \%$ (29/45) of the patients. This question was not answered by all patients, in some cases because patients would not undergo an examination on a conventional MR scanner at all. More details can be found in Table 1 .

\section{Discussion}

To our knowledge, this is the first study addressing the influence of self-hypnosis on claustrophobic events in patients undergoing MR examinations. The main findings of our study are $(a)$ audio-guided self-hypnosis significantly reduced claustrophobic events from 43 to $16 \%$ in high-risk patients; (b) the need for conscious sedation decreased from 16 to $2 \%$; and $(c)$ the need for time-consuming coping actions was reduced from 28 to $13 \%$ by audio-guided self-hypnosis. Selfhypnosis and the experience with MR imaging did not result in higher CLQ scores directly after imaging, and most patients $(67 \% ; 35 / 52)$ stated that they would prefer future MR imaging with support of self-hypnosis.
Table 3 Number of exams from different anatomical regions and corresponding claustrophobic event rate in both cohorts

\begin{tabular}{|c|c|c|c|c|c|c|c|c|}
\hline \multirow{3}{*}{$\begin{array}{l}\text { Examination } \\
\text { Combinations }\end{array}$} & \multicolumn{4}{|c|}{ Intervention group $(n=55)$} & \multicolumn{4}{|c|}{ Control group $(n=89)$} \\
\hline & \multicolumn{2}{|c|}{ Examinations } & \multicolumn{2}{|c|}{ Events } & \multicolumn{2}{|c|}{ Examinations } & \multicolumn{2}{|c|}{ Events } \\
\hline & 1 & $(2 \%)$ & 0 & $(-)$ & 12 & $(13 \%)$ & 6 & $(50 \%)$ \\
\hline Brain/head/neck & 15 & $(27 \%)$ & 4 & $(27 \%)$ & 35 & $(39 \%)$ & 17 & $(46 \%)$ \\
\hline Thorax & 4 & $(7 \%)$ & 1 & $(25 \%)$ & 3 & $(3 \%)$ & 1 & $(33 \%)$ \\
\hline Abdomen/pelvis & 24 & $(44 \%)$ & 4 & $(17 \%)$ & 27 & $(31 \%)$ & 11 & $(41 \%)$ \\
\hline Upper extremities & 5 & $(9 \%)$ & - & $(0 / 5)$ & 5 & $(6 \%)$ & 3 & $(60 \%)$ \\
\hline Lower extremities & 6 & $(11 \%)$ & - & $(0 / 6)$ & 7 & $(8 \%)$ & 0 & $(-)$ \\
\hline
\end{tabular}


Fig. 1 Flow chart of study inclusion in the non-hypnosis and the self-hypnosis cohorts. The cohort without self-hypnosis is a retrospectively evaluated patient population examined on the same MR scanner. In both cohorts, only the first examination was included. A total of 124 (25\%) of 494 referred patients were excluded because they did not undergo an examination for different reasons or were scheduled twice. In the control cohort, a majority of patients were excluded due to invasive procedures or general poor health. In the self-hypnosis cohort, $10(12 \%)$ of 87 patients were excluded because they refused to participate. Overall, 144 (29\%) of 494 patients were included in the analysis. Given is the number of all noted claustrophobic events. *Further analysis only includes the most severe event per patient

Table 4 Multivariate analysis for claustrophobic events in total and event subgroups. There were significantly less events in total, need for sedation and nonsedation coping actions in the intervention group compared to the controls after adjustment for gender, age and CLQ-values

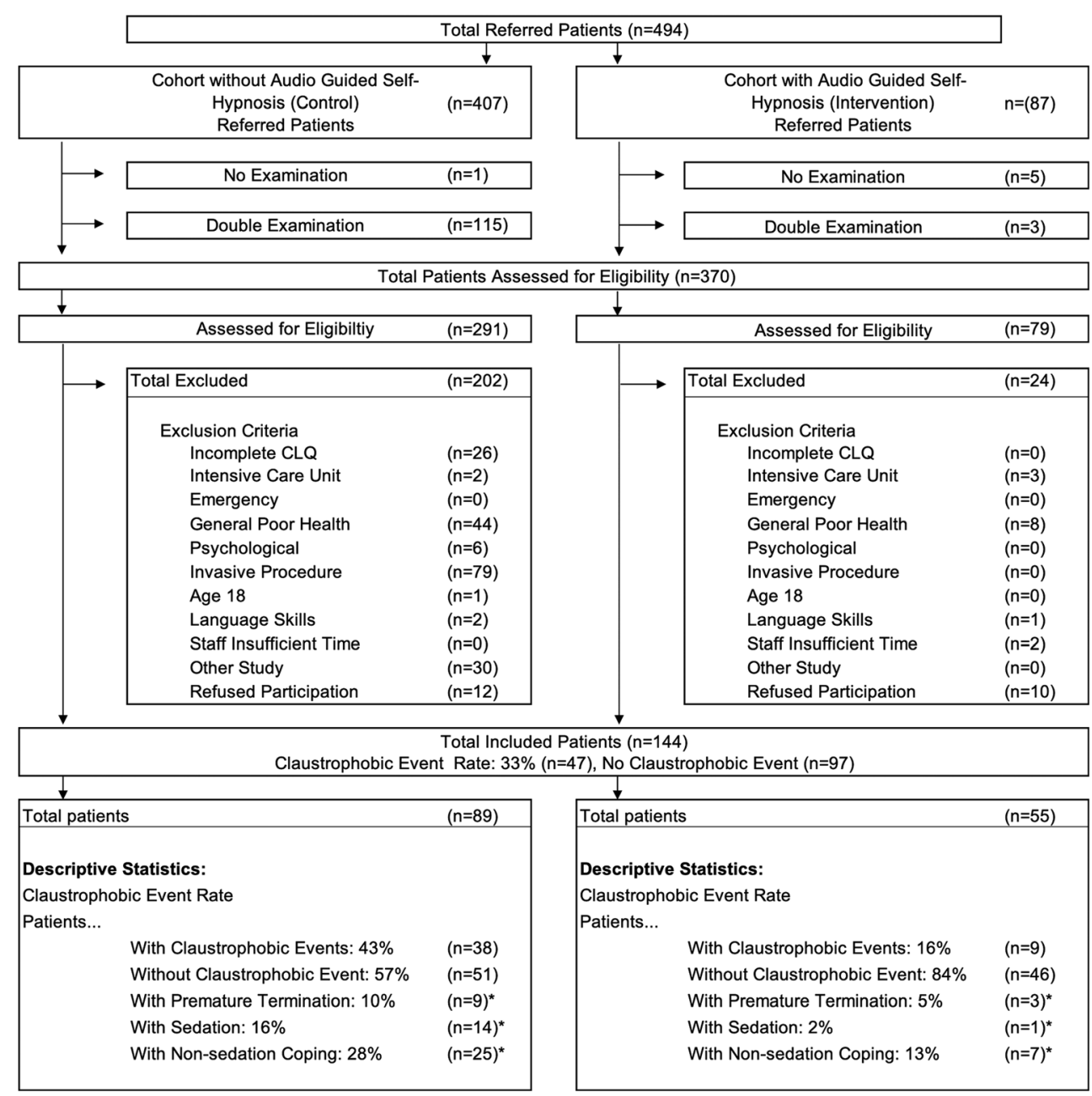

${ }^{1}$ Male vs. female

21 year

${ }^{3} 1$ point

${ }^{4}$ Yes vs. no

${ }^{5} p$ values for inclusion in forward variable selection procedure with $\mathrm{p} \_$in $=.05, \mathrm{p} \_$out $=.10$. 


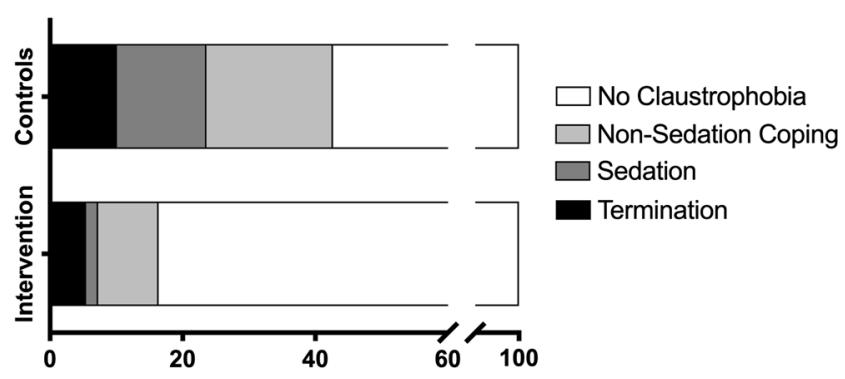

Fig. 2 Frequency of claustrophobia (percentage of patients) in intervention and control groups. Overall, there were fewer claustrophobic events, non-sedation and sedation coping actions, and premature terminations in the self-hypnosis group compared to the control group

\section{Clinical workflow}

The approach of audio-guided self-hypnosis presented here is easy to integrate into clinical workflow and saves time for the medical staff compared to individual hypnosis by an expert therapist. Self-hypnosis presented by means of an audio device can be handed over to the patient along with a short explanation when registering in the radiology department or provided on a smartphone application beforehand. The patient can use the file at his or her discretion while waiting for the examination and completing the information sheet and informed consent or use it for preparation and training at home. Our results show that selfhypnosis reduces the need for conscious sedation and non-sedation coping actions, which require extra staff and room time, medication and further patient care items as described by Bluemke et al [26]. In addition, selfhypnosis eliminates drug-related adverse events that may occur in some patients [26] and could thus be of special interest for outpatient centers, as self-hypnosis has no known negative effect on the patient's ability to drive a car after the examination.

\section{Effect of hypnosis}

Previous investigations show a positive effect of hypnosis on other medical procedures especially in terms of the need for medical sedation [19, 27-29]. Hypnosis can reduce the amount of medications needed or even completely replace benzodiazepines [22, 30-32]. Particularly, patients with comorbidities precluding sedating drug therapy or children might benefit from hypnosis [33, 34]. Self-relaxation techniques can be used by most patients referred for radiological procedures - complex scoring or assessment of hypnotic susceptibility is not required [35]. Hypnosis has thus been shown to reduce the cost of medical treatment during invasive radiologic procedures by Lang et al [36].

Some patients are still deterred by myths and misinformation about hypnosis and hypnotical trance, believing that they might lose control or experience an unwilling influence on their behavior [37]. However, we also face a growing number of patients showing a critical attitude toward drug therapy during information talks and therefore willing to use alternative treatments, which they believe to be less harmful. Therefore, self-hypnosis should be offered to patients to increase comfort and reduce the need for other coping strategies during MR.

\section{Limitations}

The type of hypnosis might not fit all patients and could lose effect compared to individual hypnosis performed by a specialist. Furthermore, we were not able to assess the entering or deepness of the hypnotic state. Nevertheless, our results show that this type of self-hypnosis has beneficial effects. A selection bias may be present as our criteria favored inclusion of patients with a positive attitude, while excluding patients with a critical attitude to hypnosis and relaxation exercises. This bias might also lead to the observed higher proportion of women in the interventional cohort. Critical patients may be more difficult to hypnotize; however, most hypnotherapists stress that "believing" in hypnosis is not mandatory for hypnotic susceptibility [38]. Unfortunately, we have not enough information to present a detailed analysis of those patients, who refused to participate. Another limitation is that we did not randomize patients but used instead a historical control cohort. Therefore, there may be effects on our results that we are not aware of, e.g., due to the slightly different distribution of exam types in both cohorts. However, we compared results for gender, age, and the CLQ scores and corrected the statistics for confounders. We did not compare self-hypnosis such as offering relaxing music or a text without suggestions. Hence, attentive listening alone might have had an effect in individual patients. Furthermore, effects on image quality were not assessed for this analysis. Finally, the audio file that we designed has a total duration of $23 \mathrm{~min}$. This might be slightly too long for daily practice; however, this can be adjusted. Furthermore, it was done by the patient alone without increased efforts by the staff.

\section{Conclusions}

Audio-guided self-hypnosis reduced claustrophobia in patients undergoing imaging in an open MR scanner with a high risk based on the claustrophobia questionnaire. Self-hypnosis appears to be a valuable tool for reducing medical sedation and the time medical staff spends on coping strategies. Further studies are warranted to address the effectiveness of selfhypnosis compared to standard care and investigate its effect in a large, randomized, and sham-controlled trial. 
Supplementary Information The online version contains supplementary material available at https://doi.org/10.1007/s00330-021-07887-w.

Acknowledgements Open Access funding enabled and organized by Projekt DEAL. We would like to thank the patients who went on this exciting journey with us and the technicians and physicians at Charité who conducted the MRI examinations. Our special thanks also go to Mrs. Christine Germershausen who assisted in setting up the study and organizing the MP3 players for the audio-guided self-hypnosis.

Funding Funding was received from Deutsche Forschungsgemeinschaft (DFG) to cover travel costs.

\section{Declarations}

Guarantor The scientific guarantor of this publication is Dr. rer. medic. Adriane Napp.

Conflict of interest The authors of this manuscript declare relationships with the following companies: activities not related to the present article: received institutional grants from Siemens Medical Solutions, GE Healthcare, Toshiba Medical Systems, and Philips Medical Systems

Statistics and biometry Prof. Dr. Peter Martus kindly provided statistical advice for this manuscript.

Informed consent Only if the study is on human subjects:

Written informed consent was obtained from all subjects (patients) who were offered audio-guided self-hypnosis in the study.

For the retrospective cohort, written informed consent was waved by the institutional review board, since the claustrophobia questionnaire is a validated instrument. The patients were informed by the investigators and gave oral consent.

Ethical approval Institutional Review Board approval was obtained.

\section{Methodology}

- prospective study with comparison to retrospective cohort

- performed at one institution

Open Access This article is licensed under a Creative Commons Attribution 4.0 International License, which permits use, sharing, adaptation, distribution and reproduction in any medium or format, as long as you give appropriate credit to the original author(s) and the source, provide a link to the Creative Commons licence, and indicate if changes were made. The images or other third party material in this article are included in the article's Creative Commons licence, unless indicated otherwise in a credit line to the material. If material is not included in the article's Creative Commons licence and your intended use is not permitted by statutory regulation or exceeds the permitted use, you will need to obtain permission directly from the copyright holder. To view a copy of this licence, visit http://creativecommons.org/licenses/by/4.0/.

\section{References}

1. Enders J, Zimmermann E, Rief M et al (2011) Reduction of claustrophobia with short-bore versus open magnetic resonance imaging: a randomized controlled trial. PLoS One 6:e23494. https://doi. org/10.1371/journal.pone.0023494
2. Dewey M, Schink T, Dewey CF (2007) Claustrophobia during magnetic resonance imaging: cohort study in over 55,000 patients. J Magn Reson Imaging 26:1322-1327. https://doi.org/10.1002/ jmri.21147

3. Russo RJ, Costa HS, Silva PD et al (2017) Assessing the risks associated with MRI in patients with a pacemaker or defibrillator. N Engl J Med 376:755-764. https://doi.org/10.1056/ NEJMoa1603265

4. Roguin A, Zviman MM, Meininger GR et al (2004) Modern pacemaker and implantable cardioverter/defibrillator systems can be magnetic resonance imaging safe: in vitro and in vivo assessment of safety and function at $1.5 \mathrm{~T}$. Circulation 110:475-482

5. Katz RC, Wilson L, Frazer N (1994) Anxiety and its determinants in patients undergoing magnetic resonance imaging. J Behav Ther Exp Psychiatry 25:131-134

6. Tornqvist E, Mansson A, Larsson EM, Hallstrom I (2006) Impact of extended written information on patient anxiety and image motion artifacts during magnetic resonance imaging. Acta Radiol 47: 474-480

7. Katznelson R, Djaiani GN, Minkovich L et al (2008) Prevalence of claustrophobia and magnetic resonance imaging after coronary artery bypass graft surgery. Neuropsychiatr Dis Treat 4:487-493

8. Törnqvist E, Månsson A, Larsson EM, Hallström I (2006) It's like being in another world-patients' lived experience of magnetic resonance imaging. J Clin Nurs 15:954-961. https://doi.org/10.1111/j. 1365-2702.2006.01499.x

9. Brand KP (1994) How well is your patient prepared for an MRI? An insider's perspective. Cancer Nurs 17:512-515

10. Harris LM, Cumming SR, Menzies RG (2004) Predicting anxiety in magnetic resonance imaging scans. Int J Behav Med 11:1-7. https://doi.org/10.1207/s15327558ijbm1101_1

11. Napp AE, Enders J, Roehle R et al (2017) Analysis and Prediction of Claustrophobia during MR Imaging with the Claustrophobia Questionnaire: An Observational Prospective 18-month SingleCenter Study of 6500 Patients. Radiology 283:148-157. https:// doi.org/10.1148/radiol.2016160476

12. Richa F, Chalhoub V, Dagher C, Yazbeck PH (2015) Dexmedetomidine sedation for a claustrophobic patient with obstructive sleep apnea undergoing magnetic resonance imaging. J Clin Anesth. https://doi.org/10.1016/j.jclinane.2015.05.017

13. Delgado J, Toro R, Rascovsky S et al (2015) Chloral hydrate in pediatric magnetic resonance imaging: evaluation of a 10-year sedation experience administered by radiologists. Pediatr Radiol 45: 108-114. https://doi.org/10.1007/s00247-014-3091-0

14. Tith S, Lalwani K, Fu R (2012) Complications of three deep sedation methods for magnetic resonance imaging. J Anaesthesiol Clin Pharmacol 28:178-184. https://doi.org/10.4103/0970-9185.94837

15. Middelkamp JE, Forster BB, Keogh C, Lennox P, Mayson K (2009) Evaluation of adult outpatient magnetic resonance imaging sedation practices: are patients being sedated optimally? Can Assoc Radiol J 60:190-195. https://doi.org/10.1016/j.carj.2009.06.002

16. Viggiano MP, Giganti F, Rossi A et al (2015) Impact of psychological interventions on reducing anxiety, fear and the need for sedation in children undergoing magnetic resonance imaging. Pediatr Rep 7:5682. https://doi.org/10.4081/pr.2015.5682

17. Munn Z, Jordan Z (2013) Interventions to reduce anxiety, distress and the need for sedation in adult patients undergoing magnetic resonance imaging: a systematic review. Int J Evid Based Healthc 11:265-274. https://doi.org/10.1111/1744-1609.12045

18. Schupp CJ, Berbaum K, Berbaum M, Lang EV (2005) Pain and anxiety during interventional radiologic procedures: effect of patients' state anxiety at baseline and modulation by nonpharmacologic analgesia adjuncts. J Vasc Interv Radiol 16: 1585-1592. https://doi.org/10.1097/01.rvi.0000185418.82287.72

19. Lang EV, Berbaum KS, Pauker SG et al (2008) Beneficial effects of hypnosis and adverse effects of empathic attention during 
percutaneous tumor treatment: when being nice does not suffice. J Vasc Interv Radiol 19:897-905. https://doi.org/10.1016/j.jvir.2008. 01.027

20. Flory N, Salazar GM, Lang EV (2007) Hypnosis for acute distress management during medical procedures. Int J Clin Exp Hypn 55: 303-317. https://doi.org/10.1080/00207140701338670

21. Lutgendorf SK, Lang EV, Berbaum KS et al (2007) Effects of age on responsiveness to adjunct hypnotic analgesia during invasive medical procedures. Psychosom Med 69:191-199. https://doi.org/ 10.1097/PSY.0b013e31803133ea

22. Lang EV, Berbaum KS, Faintuch S et al (2006) Adjunctive selfhypnotic relaxation for outpatient medical procedures: a prospective randomized trial with women undergoing large core breast biopsy. Pain 126:155-164. https://doi.org/10.1016/j.pain.2006.06. 035

23. Halsband U, Wolf TG (2019) Functional changes in brain activity after hypnosis: neurobiological mechanisms and application to patients with a specific phobia-Limitations and Future Directions. Int J Clin Exp Hypn 67:449-474. https://doi.org/10.1080/00207144. 2019.1650551

24. Radomsky AS, Rachman S, Thordarson DS, McIsaac HK, Teachman BA (2001) The claustrophobia questionnaire. J Anxiety Disord 15:287-297

25. American Society of Anesthesiologists Task Force on Sedation and Analgesia by Non-Anesthesiologists (2002) Practice guidelines for sedation and analgesia by non-anesthesiologists. Anesthesiology 96:1004-1017

26. Bluemke DA, Breiter SN (2000) Sedation procedures in MR imaging: safety, effectiveness, and nursing effect on examinations. Radiology 216:645-652. https://doi.org/10.1148/radiology.216.3. r00se 45645

27. Iserson KV (2014) An hypnotic suggestion: review of hypnosis for clinical emergency care. J Emerg Med 46:588-596. https://doi.org/ 10.1016/j.jemermed.2013.09.024

28. Dominguez-Ortega L, Rodriguez-Munoz S (2010) The effectiveness of clinical hypnosis in the digestive endoscopy: a multiple case report. Am J Clin Hypn 53:101-107. https://doi.org/10.1080/ 00029157.2010 .10404332
29. Simon EP (1999) Hypnosis using a communication device to increase magnetic resonance imaging tolerance with a claustrophobic patient. Mil Med 164:71-72

30. Eren G, Dogan Y, Demir G et al (2015) Hypnosis for sedation in transesophageal echocardiography: a comparison with midazolam. Ann Saudi Med 35:58-63. https://doi.org/10.5144/0256-4947. 2015.58

31. Zahid MF (2015) Methods of reducing pain during bone marrow biopsy: a narrative review. Ann Palliat Med 4:184-193. https://doi. org/10.3978/j.issn.2224-5820.2015.09.02

32. Friday PJ, Kubal WS (1990) Magnetic resonance imaging: improved patient tolerance utilizing medical hypnosis. Am J Clin Hypn 33:80-84. https://doi.org/10.1080/00029157.1990.10402908

33. Kiss G, Butler J (2011) Hypnosis for cataract surgery in an American Society of Anesthesiologists physical status IV patient. Anaesth Intensive Care 39:1139-1141

34. Bayat A, Ramaiah R, Bhananker SM (2010) Analgesia and sedation for children undergoing burn wound care. Expert Rev Neurother 10:1747-1759. https://doi.org/10.1586/ern.10.158

35. Fick LJ, Lang EV, Logan HL, Lutgendorf S, Benotsch EG (1999) Imagery content during nonpharmacologic analgesia in the procedure suite: where your patients would rather be. Acad Radiol 6: 457-463

36. Lang EV, Rosen MP (2002) Cost analysis of adjunct hypnosis with sedation during outpatient interventional radiologic procedures. Radiology 222:375-382. https://doi.org/10.1148/radiol. 2222010528

37. Meyerson J (2014) The myth of hypnosis: the need for remythification. Int J Clin Exp Hypn 62:378-393. https://doi.org/ 10.1080/00207144.2014.901090

38. Coe WC, Steen P (1981) Examining the relationship between believing one will respond to hypnotic suggestions and hypnotic responsiveness. Am J Clin Hypn 24:22-32. https://doi.org/10.1080/ 00029157.1981 .10403280

Publisher's note Springer Nature remains neutral with regard to jurisdictional claims in published maps and institutional affiliations. 